\title{
Coherent Vortices Properties of Partially Coherent Elegant Laguerre-Gaussian Beams in the Free Space
}

\author{
Miao Dong*, Yuanjie Yang \\ School of Physics, University of Electronic Science and Technology of China, Chengdu, China \\ Email: *dongmiao@uestc.edu.cn
}

How to cite this paper: Dong, M. and Yang, Y.J. (2020) Coherent Vortices Properties of Partially Coherent Elegant Laguerre-Gaussian Beams in the Free Space. Optics and Photonics Journal, 10, 159-166. https://doi.org/10.4236/opj.2020.106017

Received: June 5, 2020

Accepted: June 26, 2020

Published: June 29, 2020

\begin{abstract}
The analytical expression of the cross-spectral density of partially coherent elegant Laguerre-Gaussian beams propagating in free space has been derived. The coherence vortex properties of such beams have been investigated. The effect of the beam parameters, including the topological charge, radial mode index and coherence length on the coherent vortex, is analyzed. The results show that the higher order (th) of coherent vortices split to th first order of coherent vortex. New coherent vortices of opposite sign appear, and then pairs of coherent vortices form. The propagation distance and coherence length affect the generation of coherent vortices, while the radial mode index doesn't affect the coherent vortices. These results might be helpful for potential application of such beams in optical communication.
\end{abstract}

\section{Keywords}

Coherent Vortices, Topological Charge, Coherence Singularities

\section{Introduction}

Vortex beam has been widely studied due to its special properties [1] [2], such as the helical phase structure, the phase singularity, the dark hollow area and the orbital angular momentum. These special properties make it suitable for applying in optical communication [3], optical manipulation [4] and quantum information [5]. Recently, the study of optical vortex has been extended to partially coherent fields. According to the partial coherence theory [6], partially coherent beams don't possess a well-defined phase and are characterized by statistical properties. Therefore, the characteristics of vortex beam vanish under low coherence case. For example, partially coherent vortex beam does not possess the 
typical region of zero intensity, and hence does not possess any obvious phase singularities [7]. This makes it difficult for studying vortex beam with low coherence. In 2003, Schouten et al. [8] examined two-point correlation function of partially coherent beams using the Young's interference experiment. It was shown that there exist phase singularities of the spectral degree of coherence of the field at pairs of points. Such phase singularities are termed as "coherence singularities". Further investigation showed that the phase of spectral degree of coherence possesses a vortex structure around these singular points [9]. The new term "coherence vortices" is used to refer to them. These findings reveal that the vortex structure of partially coherent vortex beam is just "hidden" in the correlation function. Since then, these new physical phenomena and concepts arise a lot of interest [10]-[15]. Many investigations revealed that there is an intimate relationship between optical vortices produced by a coherent field and the corresponding coherent vortices produced by a partially coherent beam [16]. As the coherence length decreases, the optical vortex can evolve into coherent vortex. Recently, researchers group showed that the phase distribution of the cross spectral density of partially coherent vortex beams possesses rich information [17] [18]. It was shown that the number of coherent vortex is equal to the topological charge. It provides a way to measure the topological charge of partially coherent vortex beams. The results can also apply to other fields, such as information transmission and imaging.

On the other hand, partially coherent elegant Laguerre-Gaussian (eLG) beams, as the natural extension of partially coherent standard Laguerre-Gaussian (SLG) beams, represent a typical kind of partially coherent vortex beams [19]. They have been widely investigated. For example, the propagation properties of partially coherent ELG beams are less affected by the turbulence [20] [21], and the spreading of such beam is slower through the free space and the turbulent atmosphere. The coherence properties of partially coherent eLG beam are quite different from corresponding SLG beam [22]. As far as we know, the coherent vortex properties of partially coherent ELG beams have not been studied. In the paper, we have derived the analytical expression of the cross spectral density function of a partially coherent eLG beam propagating in free space. The effect of topological charge, radial mode index and coherence length on the coherent vortices have been analyzed.

\section{Coherence Vortex of Partially Coherent eLG Beams}

Consider the electric field distribution of an eLG beam at the source plane $(z=$ 0) [19]:

$$
E(\rho, \theta ; 0)=\left(\frac{\rho}{w_{0}}\right)^{|l|} L_{p}^{|l|}\left(\frac{\rho^{2}}{w_{0}^{2}}\right) \exp \left(-\frac{\rho^{2}}{w_{0}^{2}}\right) \exp (-i l \theta)
$$

where $\rho$ and $\theta$ are the radial and azimuthal coordinates, respectively, $w_{0}$ is the beam waist width of the fundamental Gaussian mode, $L_{p}^{l}$ is the Laguerre poly- 
nomial with the radial mode index $p$ and topological charge || .

Based on the partial coherence theory, the cross spectral density of a partially coherent beam at the source plane can be written as [6]:

$$
W\left(\boldsymbol{\rho}, \boldsymbol{\rho}_{2}, 0\right)=\left\langle E^{*}\left(\boldsymbol{\rho}_{1}, 0\right) E\left(\boldsymbol{\rho}_{2}, 0\right)\right\rangle
$$

where $\langle\cdot\rangle$ denotes an ensemble average, $\boldsymbol{\rho}_{i}=\left(\rho_{i}, \theta_{i}\right)$ is a position vector of a point in the source plane. Here we assume a partially coherent light source in the initial plane with field correlation properties described by a Gaussian-Schell correlator:

$$
C\left(\rho_{1}, \rho_{2}, 0\right)=\exp \left(-\frac{\left(\rho_{1}-\rho_{2}\right)^{2}}{\delta^{2}}\right)
$$

where $\delta$ is the coherence length.

Substituting Equation (1) and Equation (3) into Equation (2), the cross spectral density of a partially coherent eLG beam at the source plane can be expressed as:

$$
\begin{aligned}
& W\left(\boldsymbol{\rho}, \boldsymbol{\rho}_{2}, 0\right)=\left(\frac{\rho_{1} \rho_{2}}{w_{0}^{2}}\right)^{|l|} L_{p}^{l}\left(\frac{\rho_{1}^{2}}{w_{0}^{2}}\right) L_{p}^{l}\left(\frac{\rho_{2}^{2}}{w_{0}^{2}}\right) \exp \left(-\frac{\rho_{1}^{2}+\rho_{2}^{2}}{w_{0}^{2}}\right) \\
& \exp \left(i l\left(\theta_{1}-\theta_{2}\right)\right) \exp \left(-\frac{\left(\rho_{1}-\rho_{2}\right)^{2}}{\delta^{2}}\right)
\end{aligned}
$$

In the framework of the paraxial approximation, the cross spectral density of such beam propagating in free space can be expressed [20]:

$$
\begin{aligned}
& W\left(\boldsymbol{\rho}_{1}, \boldsymbol{\rho}_{2}, z\right)=\left(\frac{k}{2 \pi z}\right)^{2} \iint_{\infty} W\left(\boldsymbol{\rho}_{0}, \boldsymbol{\rho}_{20}, 0\right) \\
& \times \exp \left\{-\frac{i k}{2 z}\left[\left(\boldsymbol{\rho}-\boldsymbol{\rho}_{0}\right)^{2}-\left(\boldsymbol{\rho}-\boldsymbol{\rho}_{20}\right)^{2}\right]\right\} d^{2} \boldsymbol{\rho}_{0} d^{2} \boldsymbol{\rho}_{20}
\end{aligned}
$$

where $k=2 \pi / \lambda$ is wavenumber; $z$ is the distance in free space.

The spectral degree of coherence is defined as [9]:

$$
\mu\left(\rho_{1}, \rho_{2}\right)=\frac{W\left(\rho, \rho_{2}\right)}{\sqrt{S(\rho)} \sqrt{S\left(\rho_{2}\right)}}
$$

where $S(\rho)=W(\rho, \rho)$ is the spectral density. Since the spectral density is non-zero for the partially coherent beam, the position of coherence vortices at pairs of points $\left(x_{1}, y_{1}\right)$ and $\left(x_{2}, y_{2}\right)$ can be expressed as:

$$
\begin{aligned}
& \operatorname{Re}\left[W\left(\boldsymbol{\rho}, \boldsymbol{\rho}_{2}\right)\right]=0 \\
& \operatorname{Im}\left[W\left(\boldsymbol{\rho}, \boldsymbol{\rho}_{2}\right)\right]=0
\end{aligned}
$$

where $\operatorname{Re}$, Im denote the real and imaginary parts, respectively.

\section{Numerical Simulation}

Based on equations above, we can investigate the phase distribution of the cross-spectral density of partially coherent elegant LG beams in free space. The 
parameters are set as: $\lambda=532 \mathrm{~nm}, w_{0}=1 \mathrm{~mm}, \delta=1 \mathrm{~mm}, \quad r_{2}=\left(0.5 w_{0}, 0\right)$, $R=\pi w_{0}^{2} / \lambda$ is Rayleigh distance.

Figure 1 shows the effect of the topological charge 1 on coherent vortices of partially coherent eLG beams with radial mode index $p=1$ at different propagation distances. From Figure 1(a), we can see that there is phase singularity and a circular dislocation at the distance $z=0$. The phase around singularity clockwise increases $2 \pi$, which corresponds to the coherent vortex with topological charge of " +1 ". The position of coherent vortex is same as that of optical vortex. With increasing propagation distance, the phase singularity remains stable, however the circular dislocation becomes unclearly. The contour lines of phase become curve and clockwise rotate. When the propagation distance further increases, a new coherent vortex with topological charge of “-1" appears. Thus, a pair of coherent vortices forms and the total angular momentum becomes zero. When the topological charge $l>1$, we can see that the phase increases $2 \pi l$ around phase singularity at $\mathrm{z}=0$. The coherent vortices of high order (th) are not stable, split to th first order coherent vortex. The corresponding coherent vortices of opposite sign of topological charge forms. Thus, the total angular momentum becomes zero. Particularly, we note that the position of the coherent vortex (No.1) always exists and remains stable while the position of other coherent vortices changes during propagation. We can see that is due to the fact that the coherent vortex of No.1 is inherent and created by vortex beam [23]. Other coherent vortices are produced by vortex beam propagating through free space.

Figure 2 gives the effect of radial index $p$ on the coherent vortices of partially coherent eLG beams with topological charge $l=1$. The other parameters are same as those in Figure 1. We can see that the number of the circular dislocation

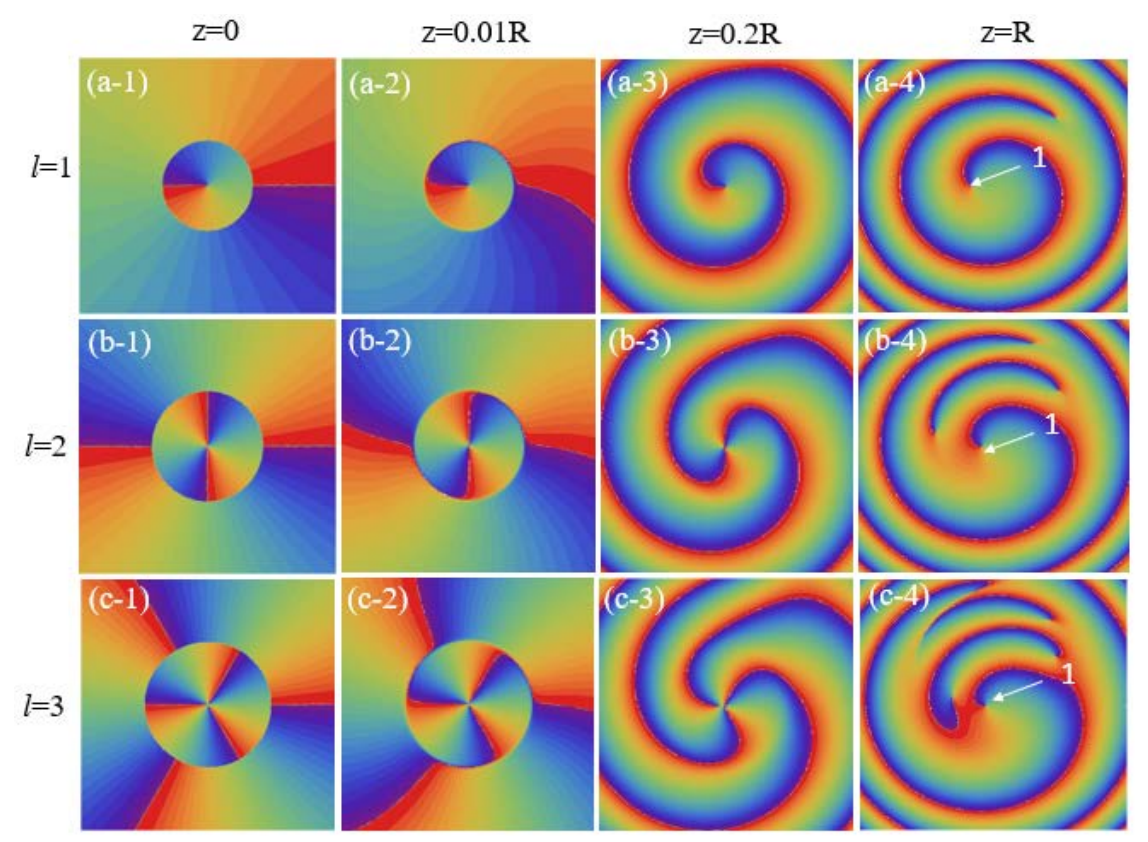

Figure 1. The effect of the topological charge $l$ on the coherent vortex at different propagation distance. (a) $I=1$; (b) $l=2$; (c) $l=3$. 
represents the value of radial mode index $p$. With the propagation distance increases, the circular dislocation becomes not clearly. When the beam arrives at the Rayleigh distance, the information of circular dislocation disappears. However, the vortex structure and phase singularity still exist. We can conclude that the radial mode index doesn't affect the coherent vortices.

Figure 3 shows the effect of the coherence length on coherence vortex of such

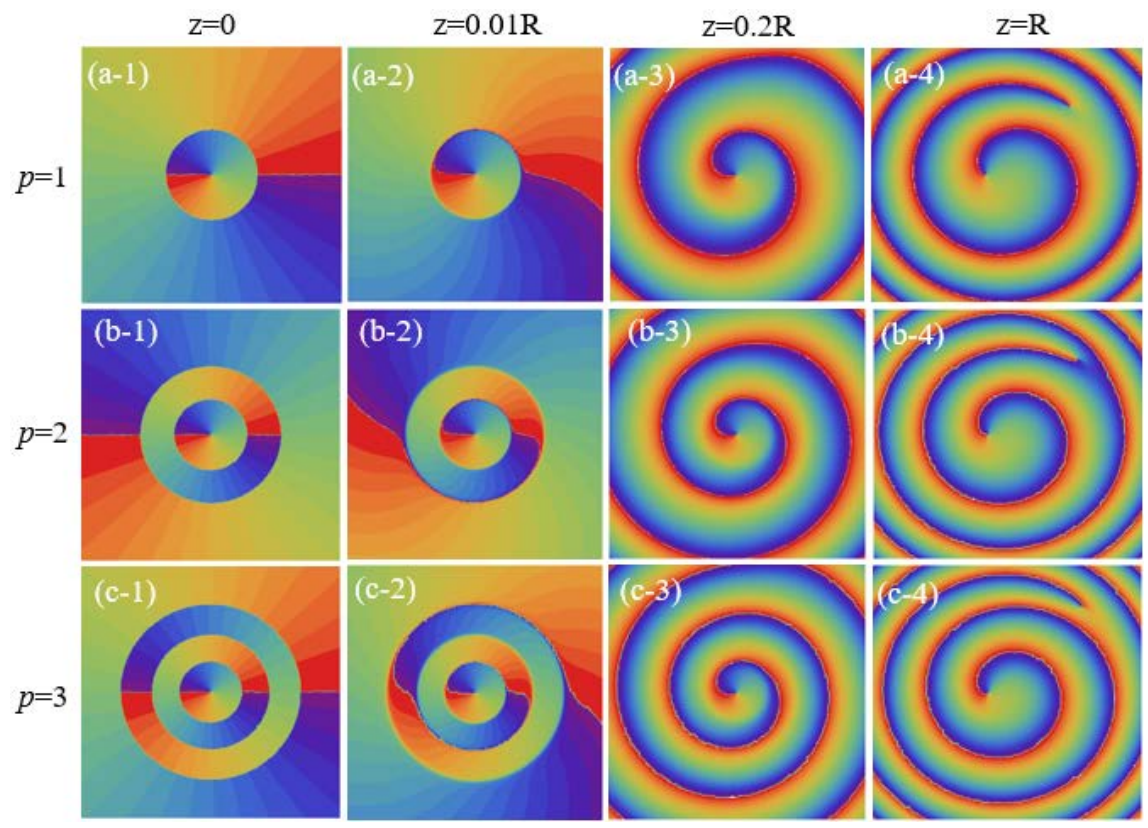

Figure 2. The effect of the radial mode index $p$ on coherent vortices at different distance. (a) $p=1$; (b) $p=2$; (c) $p=3$.

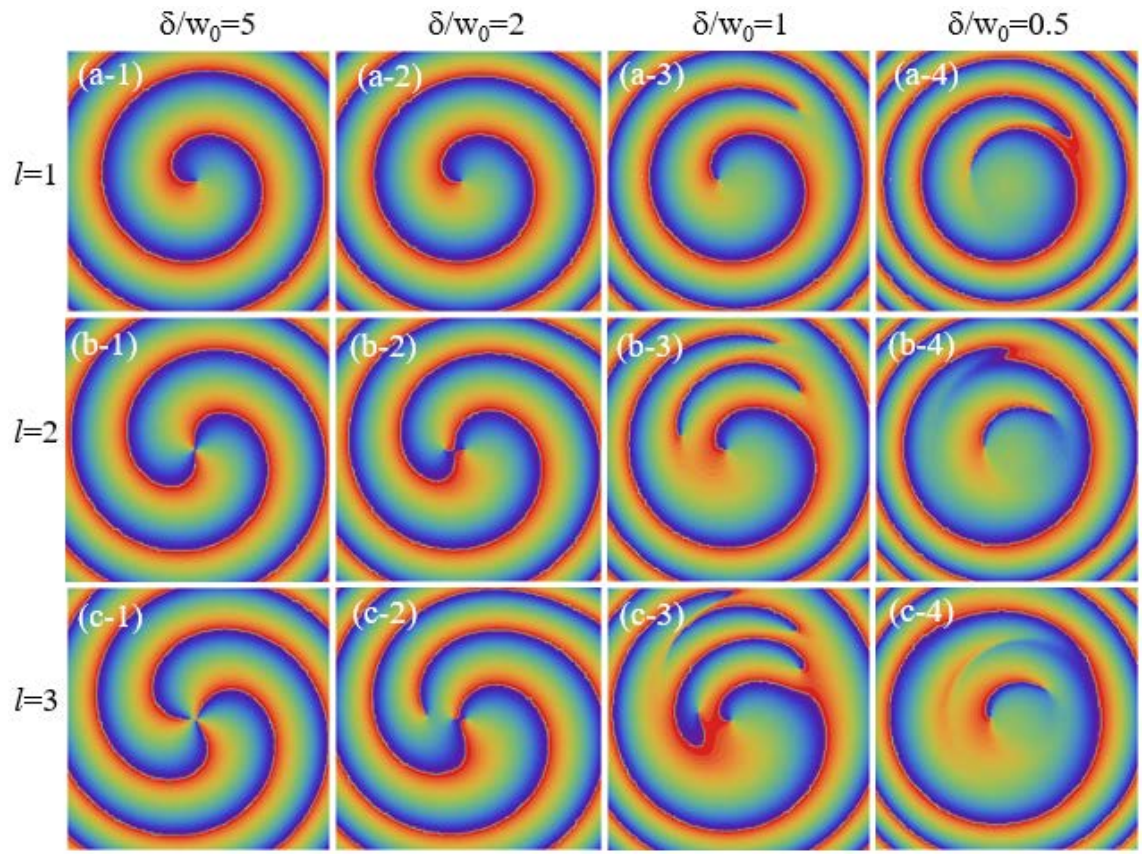

Figure 3. The effect of coherence length on the coherent vortices at Rayleigh distance. (a) $l=1, p=1$; (b) $l=2, p=1$; (c) $l=3, p=1$. 
a beam with radial index $\mathrm{p}=1$ in the Rayleigh distance. The other parameters are same as those in Figure 1. We can see that there isn't any radial mode index information. Under the high coherent case, the position of coherence vortex is same as that of optical vortex. As the coherence length decreases, the opposite sign of coherence vortex appears from infinity, and the pair of coherence vortices moves closer. The coherent vortices of higher order (th) begin to split and become th first order coherent vortex. The corresponding of coherent vortex of opposite sign of topological charge begins to appear. When the coherence further decreases, the pairs of coherence vortex move closer and begin to vanish.

\section{Conclusion}

The analytical expression of the cross-spectral density function of partially coherent elegant Laguerre-Gaussian beams propagating in free space has been derived. According to the definition of coherent vortices, the coherent vortices properties of such a beam propagating in free space are investigated. The results show that the distance and the coherence length affect the propagation properties of the coherent vortex. When the propagation distance increases or the coherence length decreases, the coherent vortices of higher order (th) split to th first order of coherent vortex, then the corresponding coherent vortex of opposite sign appears. The pairs of coherent vortices form. During propagation, the radial mode index disappears and it doesn't affect the coherent vortices properties. These finding can help to explore such beams in potential application in optical communication.

\section{Conflicts of Interest}

The authors declare no conflicts of interest regarding the publication of this paper.

\section{References}

[1] Allen, L., Beijersbergen, M.W., Spreeuw, R.J.C. and Woerdman, J.P. (1992) Orbital Angular Momentum of Light and the Transformation of Laguerre-Gaussian Laser Modes. Physical Review A, 45, 8185-8189.

http://link.aps.org/doi/10.1103/PhysRevA.45.8185 https://doi.org/10.1103/PhysRevA.45.8185

[2] Alison, M.Y. and Miles, J.P. (2011) Orbital Angular Momentum: Origins, Behavior and Applications. Advances in Optics and Photonics, 3, 161-204. https://doi.org/10.1364/AOP.3.000161

[3] Wang, J., Yang, J.Y., Fazal, I.M., et al. (2012) Terabit Free-Space Data Transmission Employing Orbital Angular Momentum Multiplexing. Nature Photonics, 6, 488-496. https://doi.org/10.1038/nphoton.2012.138

[4] He, H., Friese, M.E.J., Heckenberg, N.R. and Rubinsztein-Dunlop, H. (1995) Direct Observation of Transfer of Angular Momentum to Absorptive Particles from a Laser Beam with a Phase Singularity. Physical Review Letters, 75, 826-829. https://doi.org/10.1103/PhysRevLett.75.826

[5] Vaziri, A., Pan, J.W., Jennewein, T., et al. (2003) Concentration of Higher Dimen- 
sional Entanglement: Qutrits of Photon Orbital Angular Momentum. Physical Review Letters, 91, Article ID: 227902. https://doi.org/10.1103/PhysRevLett.91.227902

[6] Mandel, L. and Wolf, E. (1995) Optical Coherence and Quantum Optics. Cambridge University Press, New York. https://doi.org/10.1017/CBO9781139644105

[7] Gbur, G., Visser, T.D. and Wolf, E. (2004) "Hidden" Singularities in Partially Coherent Wavefields. Journal of Optics A-Pure and Applied Optics, 6, 5239-5242. https://doi.org/10.1088/1464-4258/6/5/017

[8] Schouten, H.F., Gbur, G., Visser, T.D., et al. (2003) Phase Singularities of the Coherence Functions in Young's Interference Pattern. Optics Letters, 28, 968-970. https://doi.org/10.1364/OL.28.000968

[9] Gbur, G. and Visser, T.D. (2003) Coherence Vortices in Partially Coherent Beams. Optics Communications, 222, 117-125. https://doi.org/10.1016/S0030-4018(03)01606-7

[10] Wang, W., Duan, Z.H., Hanson, S.G., Miyamoto, Y. and Takedas, M. (2006) Experimental Study of Coherence Vortices: Local Properties of Phase Singularities in a Spatial Coherence Function. Physical Review Letters, 96, Article ID: 073902. https://doi.org/10.1103/PhysRevLett.96.073902

[11] Li, J.H. and Lü, B.D. (2009) Propagation of Gaussian Schell-Model Vortex Beams through Atmospheric Turbulence and Evolution of Coherent Vortices. Journal of Optics A-Pure and Applied Optics, 11, Article ID: 045710. https://doi.org/10.1088/1464-4258/11/4/045710

[12] Liu, Z. and Zhao, D. (2012) Coherence Vortex Properties of Partially Coherent Flat-Topped Vortex Beams. Applied. Physics B, 106, 691-700. https://doi.org/10.1007/s00340-011-4848-x

[13] Maleev, I.D. and Swartzlander, G.A.J. (2008) Propagation of Spatial Correlation Vortices. Journal of the Optical Society of America B, 25, 915-921. https://doi.org/10.1364/JOSAB.25.000915

[14] Cheng, M.J., Guo, L.X., Li, J.T., Huang, Q.Q., Cheng, Q. and Zhang, D. (2016) Propagation of an Optical Vortex Carried by a Partially Coherent Laguerre-Gaussian Beam in Turbulent Ocean. Applied Optics, 55, 4642. https://doi.org/10.1364/AO.55.004642

[15] Palacios, D.M., Maleev, I.D. Marathay, A.S. and Swartzlander, G.A.J. (2004) Spatial Correlation Singularity of a Vortex Field. Physical Review Letters, 92, Article ID: 143905. https://doi.org/10.1103/PhysRevLett.92.143905

[16] Gbur, G. (2008) Optical and Coherence Vortices and Their Relationships. Proceedings of SPIE, 7008. https://doi.org/10.1117/12.796872

[17] Lu, X., Zhao, C., Shao, Y., et al. (2019) Phase Detection of Coherence Singularities and Determination of the Topological Charge of a Partially Coherent Vortex Beam. Applied Physics Letters, 114, 201106.1-201106.5. https://doi.org/10.1063/1.5095713

[18] Zeng, J., Lu, X.Y., Liu, L.X., Zhu, X.L., Zhao, C.L. and Cai, Y.J. (2019) Simultaneous Measurement of the Radial and Azimuthal Mode Indices of a Higher-Order Partially Coherent Vortex Beam Based on Phase Detection. Optics Letter, 44, 3881-3884. https://doi.org/10.1364/OL.44.003881

[19] Wang, F., Cai, Y.J. and Korotkova, J.O. (2009) Partially Coherent Standard and Elegant Laguerre-Gaussian Beams of All Orders. Optics Express, 17, 22366-22379. https://doi.org/10.1364/OE.17.022366

[20] Xia, T., Liu, D., Dong, A., et al. (2019) Properties of Partially Coherent Elegant Laguerre-Gaussian Beam in Free Space and Oceanic Turbulence. Optik, 201, Article 
ID: 163514. https://doi.org/10.1016/j.ijleo.2019.163514

[21] Li, Y.X., Cui, Z.W., Han, Y.P. and Hui, Y.F. (2019) Channel Capacity of Orbital-Angular-Momentum-Based Wireless Communication Systems with Partially Coherent Elegant Laguerre-Gaussian Beams in Oceanic Turbulence. Journal of the Optical Society of America A, 36, 471. https://doi.org/10.1364/JOSAA.36.000471

[22] Dong, M., Lu, X.Y., Zhao, C.L. and Cai, Y.J. (2018) Measuring Topological Charge of Partially Coherent Elegant Laguerre-Gaussian Beam. Optics Express, 26, 33035. https://doi.org/10.1364/OE.26.033035

[23] Li, J., Zeng, J. and Duan, M. (2015) Classification of Coherent Vortices Creation and Distance of Topological Charge Conservation in Non-Kolmogorov Atmospheric Turbulence. Optics Express, 23, 11556. https://doi.org/10.1364/OE.23.011556 